\title{
Rice False Smut Fungus, Ustilaginoidea virens, Inhibits Pollen Germination and Degrades the Integuments of Rice Ovule
}

\author{
Wenlu $\mathrm{Li}^{\mathbf{1}^{*}}$, Luoye $\mathrm{Li}^{\mathbf{1}^{*}}$, Aiqing Feng ${ }^{2}$, Xiaoyuan $\mathrm{Zhu}^{2}$, Jianxiong $\mathrm{Li}^{{ }^{1 \#}}$ \\ ${ }^{1}$ Key Laboratory of Plant Resources Conservation and Sustainable Utilization, South China Botanical Garden, Chinese Academy of \\ Sciences, Guangzhou, China; ${ }^{2}$ Plant Protection Institute, Guangdong Academy of Agricultural Sciences, Guangzhou, China. \\ Email: \#xxli@scbg.ac.cn
}

Received October $11^{\text {th }}, 2013$; revised November $12^{\text {th }}, 2013$; accepted November $23^{\text {rd }}, 2013$

Copyright (C) 2013 Wenlu Li et al. This is an open access article distributed under the Creative Commons Attribution License, which permits unrestricted use, distribution, and reproduction in any medium, provided the original work is properly cited.

\begin{abstract}
Ustilaginoidea virens is a flower-infecting fungus that forms false smut balls in rice panicle. Rice false smut has long been considered a minor disease, but recently it occurred frequently and emerged as a major disease in rice production. In vitro co-cultivation of $U$. virens strain with young rice panicles showed that $U$. virens enters inside of spikelets from the apex and then grows downward to infect floral organs. In response to $U$. virens infection, rice host exhibits elevated ROS accumulation and enhanced callose deposition. The secreted compounds of $U$. virens can suppress rice pollen germination. Examination of sectioning slides of freshly collected smut balls demonstrated that both pistil and stamens of rice flower are infected by $U$. virens, hyphae degraded the contents of the pollen cells, and also invaded the filaments. In addition, $U$. virens entered rice ovary through the thin-walled papillary cells of the stigma, then decomposed the integuments and infected the ovary. The invaded pathogen could not penetrate the epidermis and other layers of the ovary. Transverse section of the pedicel just below the smut balls showed that there were no fungal hyphae observed in the vascular bundles of the pedicel, implicating that $U$. virens is not a systemic flower-infecting fungus.
\end{abstract}

Keywords: Flower-Infecting Fungi; Integuments; Pollen Germination; Oryza sativa; Rice False Smut

\section{Introduction}

Rice false smut is a severe and widespread disease in major rice-growing areas of Asia, Africa and America [1-4]. It has been in the Unite States since at least 1906 [5]. Since that time, the disease has been sporadic and light in the US for many years. In 1997, however, high incidence of rice false smut was reported in Arkansas and Louisiana. The false smut was present on many germplasms at the Rice Research Station and was observed on commercial cultivars in growers' fields. The disease incidence ranged from $1 \%$ to $15 \%$ of tillers infected, with at least two to three spore balls per infected panicle [6]. In China, rice false smut has long been considered a minor disease in rice production, and farmers even called smut balls the harvest fruits, meaning the harbinger of a big harvest for rice yield. Recently, with the wide use of

\footnotetext{
*These authors contributed equally to this work

"Corresponding author.
}

high-yielding varieties and heavy application of nitrogen fertilizer in rice cultivation, rice false smut has emerged as the most devastating grain disease in rice production $[1,7]$.

A successful infection is indicated by the appearance of smut balls in rice panicle, which usually takes about 20 days after the infection to occur. Rice false smut disease causes yield loss and reduces grain quality. The yield losses have been attributed to not only the smut ball incidence but also the chaffiness and reduction in 1000grain weight [8], and the rice grain quality is reduced much by fungal contamination. Thus, the smut balls must be removed before rice sale in many countries as the rice grains that are contaminated with spores of $U$. virens cannot be parboiled, and the resulting product is discolored and toxic to humans and live stock.

The natural infection process of $U$. virens remains unclear, which hampers studies on the disease. However, several research groups have reported that the artificial 
inoculation method can be used to evaluate disease index of fungal infection in greenhouse [9-11]. The artificial inoculation experiments demonstrated that the late booting stage is the critical period for $U$. virens infection. The artificial inoculation method uses the mixture of conidia and hyphae produced in liquid culture to inject the leaf sheath of flag leaf at the late booting stage, leading to a much higher disease index and even more reproducibility than the field trial.

In contact with plants, pathogens can trigger an array of reactions deployed in plants to prevent pathogens' invasion, many of which appear to involve the production of reactive oxygen species (ROS) [12,13]. High levels of ROS are often accumulated rapidly after recognition of the pathogen $[14,15]$. ROS have been implicated in playing a vital role in plant defenses against pathogen invasion not only in direct antimicrobial roles but also in cellular signaling associated with the induction of defense gene expression [16], phytoalexin production $[17,18]$ and callose deposition $[19,20]$. The deposition of the callose at cell wall at the site of pathogen contact, and the pathogen-triggered callose response is required for resistance to microbial pathogens $[21,22]$.

The frequent occurrence of rice false smut makes it a serious problem in rice production recently, but the progress in understanding the routes of fungal infection was much slower than we expected. Knowing fungal infection process is critical for exploring the relationship between $U$. virens and rice host, also it is important to control the occurrence of the disease in rice production. Here, we monitored the early processes of fungal infection and detected the ROS levels and callose deposition in rice floral organs upon the recognition of $U$. virens infection. We also examined the invasion sites at the late stage of $U$. virens infection and provided evidence to show degradation of the integuments of ovary may be the critical strategy for $U$. virens to proliferate in the infected rice spikelets.

\section{Materials and Methods}

\subsection{Plant Materials, Fungal Growth and Inoculation Methods}

Rice variety Huangxiuzan was grown in the experimental station of Plant Protection Institute, Guangdong Academy of Agricultural Science, Guangdong Province, China. The experiment field was protected by a metal net, and there was an automatic water-spraying system underneath the net and above the field, this system sprayed mistlike water for 30 seconds per hour from 10 o'clock in the morning to 4 o'clock in the afternoon to increase humidity in the atmosphere during the infection period from artificial inoculation of rice plants with $U$. virens to emer- gence of the smut balls on rice panicles. PSB (potato and sucrose broth) medium was used to culture $U$. virens strain for inoculation. $U$. virens was cultured at $28^{\circ} \mathrm{C}$ for 10 days, the whole culture was stirred three times in a stirrer, each for $15 \mathrm{sec}$, to break down hyphae to small pieces, the resultant mixture of hyphae and spores were used to inoculate rice panicles. We used a syringe with needle to inoculate rice plants at booting stage, each plant was injected with $2-4 \mathrm{ml}$ of inoculum until the liquid flooded out from the top of leaf sheath. Smut balls appeared in rice panicles about 20 days after inoculation.

\subsection{In Vitro Infection of Rice Panicle}

$U$. virens was cultured in PSB medium at $28^{\circ} \mathrm{C}$ for 8 days, the culture was filtered with three-layer of lens papers to exclude hyphae, $U$. virens conidia were collected and adjusted to the concentration of $1 \times 10^{7}$ conidia per milliliter. Panicles at booting stage were peeled out and rinsed in the filter-treated conidia solution for 10 minutes. After the treatment, the panicles were cultured in $1 / 2 \mathrm{MS}$ medium for 5 days, spikelets were used to observe conidium germination and examine the early infection process of $U$. virens.

\subsection{Light Microscopy and Scanning Electron Microscopy}

$U$. virens-infected spikelets were opened carefully under a dissection microscope, the processes of hyphae infection of the anthers and stigma were observed under stereomicroscope (Zeiss Stemi SV11, Carl Zeiss, Germany) and corresponding images were taken. Anthers and stigma from in vitro $U$. virens-infected spikelets were pretreated and mounted to a metal stub (10 $\mathrm{mm}$ in diameter). The specimens were sputter-coated with gold particles (approximately $30 \mathrm{~nm}$ in thickness) and examined with a scanning electron microscope (JSM-6360 LV, JEOL Ltd, Japan).

\subsection{Semi-Thin Sectioning and Aniline Blue Staining}

Smut balls collected from the field were first fixed in FAA (Formaldehyde acetic acid ethanol) solution for 24 hours. After several times of dehydration and rehydration, the specimens were embedded in epoxy resin and cut with a glass knife into semi-thin sections. The semi-thin sections were carried out aniline blue staining according to the procedure described by Hood and Shew [23] with minor modification. The stained sections were placed on glass slide and examined by means of epifluorescence microscopy (Zeiss Axioplan 2, Carl Zeiss, Germany), and images were record by Zeiss AxioCam. 


\subsection{ROS Detection and Callose Deposition}

To detect ROS production on the rice flower organs during $U$. virens infection, spikelets from infected panicles and uninfected control panicles were stained with 3, 3'Diaminobenzidine tetrahydrochloride solution $(1 \mathrm{mg}$ of DAB-HCl per $1 \mathrm{~mL}$ of water) for $3 \mathrm{~h}$ at room temperature. The samples were destained in ethanol:chloroform (4:1) and then kept in the dark in $60 \%$ glycerol until examination by light microscopy.

Callose deposition was monitored by potassium hydroxide-aniline blue staining. Briefly, the materials were vacuum infiltrated in $1 \mathrm{M}$ potassium hydroxide for $2 \mathrm{~h}$ at room temperature, followed by staining with $0.05 \%(\mathrm{w} / \mathrm{v})$ aniline blue in water. The stained materials were placed in glass slides and examined by means of epifluorescence microscopy (Leica DMRBE, Switzerland).

\section{Results}

\subsection{Invasion Routes of $U$. virens at the Early Infection Stage}

We used freshly cultured $U$. virens to inoculate rice panicles at the late booting stage, the concentration of inoculum was $10^{7}$ conidia per milliliter (Figure 1(a)). At day 5 after infection, hyphae emerged and enclosed rice spikelets (Figure 1(b)). Usually, flower-infecting fungi develop hyphae on the exterior surface of florets and grow toward the openings. Rice spikelets covered by $U$. virens hyphae were opened up to examine the infection process inside the spikelet (Figure 1(c)). Hyphae entered florets through the aperture of the apex of glumes, firstly occupied the upper space of the flower organs and formed a three-dimensional network (Figure 1(d)), then grew downward and intertwined the stamens and the bifurcate feathery stigma (Figure 1(e)). We observed large amounts of hyphae on the surface of anthers and plumose stigma (Figures 1(f), (g)). Growing downward, hyphae filled the whole inside space of the floret and enclosed the ovary (Figure 1(h)). This observation is in agreement with the findings of Ashizawa et al. [24] in that they found $U$. virens invaded rice spikelets through the small gap of the spikelet apex.

\subsection{U. virens Infection Induces Elevated Levels of ROS and Enhanced Callose Deposition}

Rice panicles were collected from $U$. virens infected plants at day 3 after infection, the panicles were immersed in a solution of 3, 3'-diaminobenzidine tetrahydrochloride (DAB-HCl) for 3 hours at room temperature, after destained in ethanol:chloroform solution, rice flower organs were observed under microscope to exam-

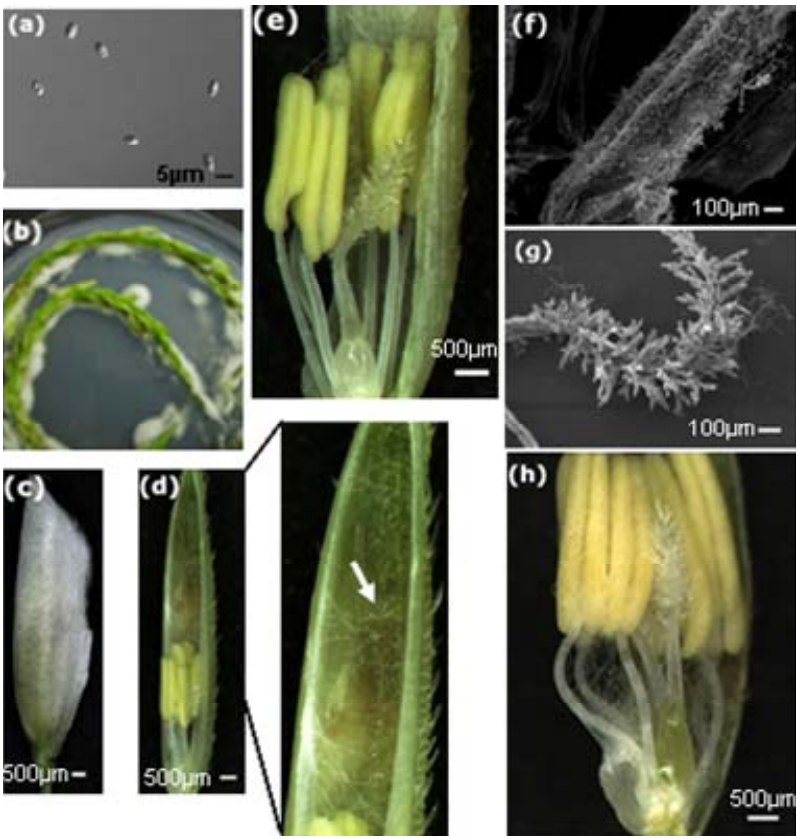

Figure 1. Invasion routes of $U$. virens in the early interaction with rice host. (a) Conidia used for in vitro inoculation of rice panicles; (b) Hyphae grow on the surface of rice panicles after co-cultivation on 1/2 MS medium; (c) A spikelet enclosed by white hyphae; (d) The spikelet in (c) was opened, showing hyphae inside the glumes and on the top of stamen, high magnification of the top of the glumes is showed on the right, white arrow indicates hyphae; (e) High magnification of the lower part of (d) showing hyphae on anthers and stigma,hyphae are not observed on the base of floral organs; (f) Scanning electron microsocopy showing hyphae on the surface of the anther; (g) Scanning electron microsocopy showing hyphae around the stigma; (h) $\mathrm{Hy}$ phae infected the base of floral organs, surrounding the anther filaments and enclosing the ovary.

ine ROS accumulation. DAB forms a brown polymerization product in the presence of ROS. Flower organs from $U$. virens-infected panicles showed enhanced brown oxidized DAB precipitates (Figures 2(a)-(c)), in contrast, the flower organs from the control plants did not exhibit brown color (Figures 2(d)-(f)). We cut the ovaries from both the $U$. virens-infected and the control plants and examined the $\mathrm{DAB}$ oxidation under microscope, the ovaries from $U$. virens-infected plants displayed more $\mathrm{DAB}$ oxidation products than those from uninfected plants (Figures 2(a), (d)), especially, the base of the infected ovary showed the highest level of oxidation, indicating more ROS produced at this site. Probably, the base of the ovary is the place where the fungus obtains water and nutrients from host plant.

The stigma from $U$. virens-infected plants showed enhanced accumulation of ROS as evidenced by the brown DAB oxidation, whereas the stigma of the control plants did not show brown color (Figures 2(b), (e)). In addi- 

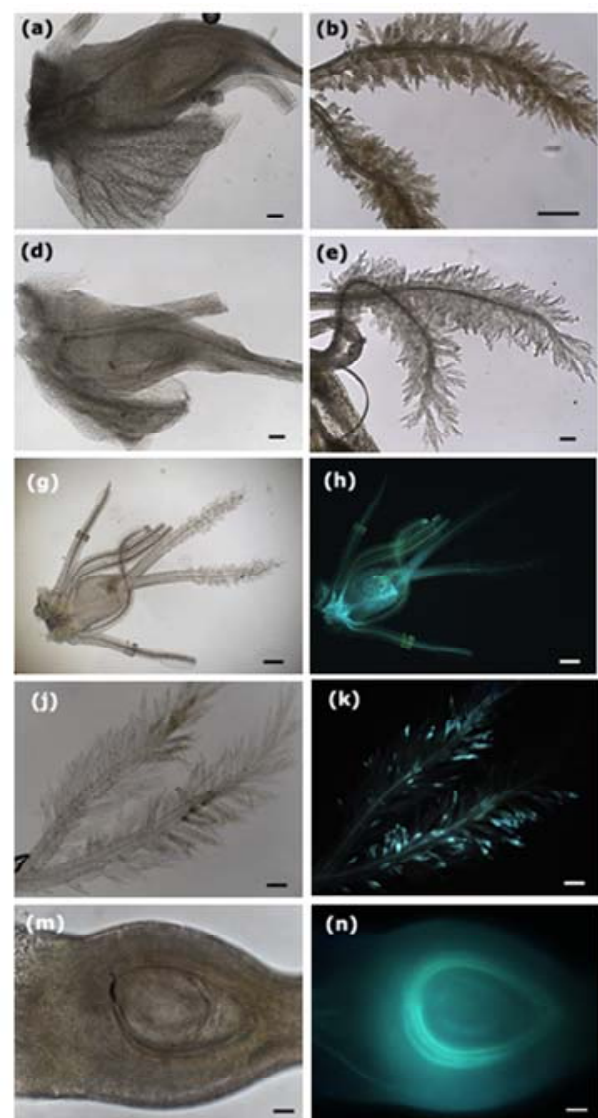

(n)
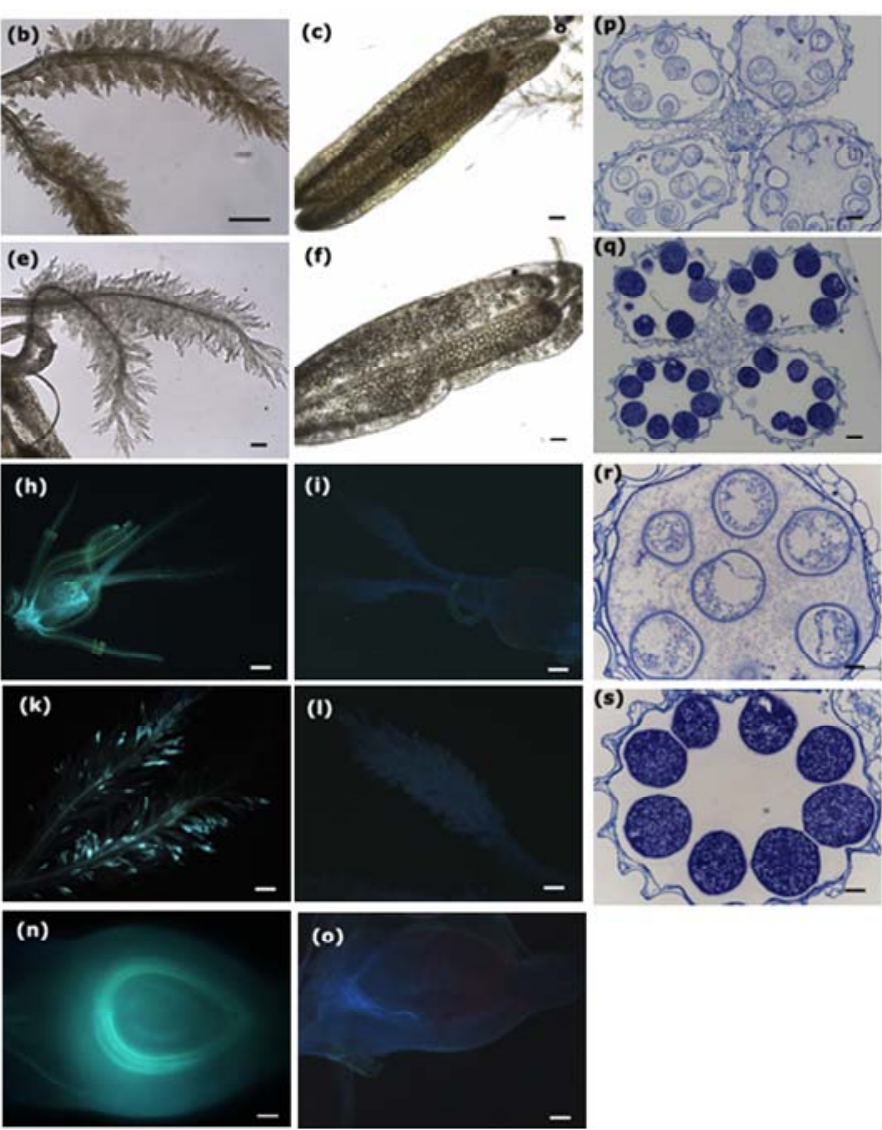

Figure 2. Infection of $U$. virens induces reactive oxygen species (ROS) accumulation, enhances callose deposition, and inhibits the development of pollen mother cells. Scale bars: (a)-(f), $100 \mu \mathrm{m}$; (g)-(o), $200 \mu \mathrm{m}$; (p)-(s), 50 $\mathrm{m}$. (a)-(c) Infection of $U$. virens induced ROS accumulation on the surfaces of the ovary, stigma and anther, respectively; (d)-(f) The ovary, stigma and anther from uninfected plants did not show ROS accumulation; (g) Rice flower from the $U$. virens-infected plant was observed under bright light of microscope; (h) The flower in (g) was observed under ultraviolet showing bright color, indicating the callose deposition on the surface of the flower; (i) The flower organ from uninfected plants was observed under ultraviolet, not showing bright color; (j) The feathery stigma from infected plant was observed under bright light of microscope; (k) The stigma in (j) was observed under ultraviolet, showing bright spots scattered on the stigma, indicating the sites of callose deposition; (l) The stigma of uninfected plant was observed under ultraviolet, not showing bright color; (m) The ovary from infected plant was cut and observed under bright light of microscope; (n) The ovary in (m) was observed under ultraviolet, showing a bright circle surrounding the nucellus of the ovary, suggesting a strong callose deposition at that position; (o) The ovary from uninfected plant was cut and observed under ultraviolet, not showing bright color; (p) Transverse section of the anthers from fungus-infected spikelets, indicating developmental inhibition of the pollen mother cells; (q) Transverse section of the anthers from control plants, showing normal development of the pollen mother cells; (r) High magnification of (p), showing plasmocytosis of the pollen mother cells; (s) High magnification of (q), showing normal shape of pollen mother cells.

tion, the anthers from $U$. virens-infected plants had higher levels of DAB oxidation than those of the uninfected plants (Figures 2(c), (f)), indicating that $U$. virens infection induced high levels of ROS accumulation at the places it invaded.

Callose plays important roles in response to multiple biotic and abiotic stresses, and can be induced upon the recognition of pathogen infection. We collected rice panicles at day 5 after $U$. virens inoculation to clarify callose deposition. Flower organs from $U$. virens infected plants were first recorded under bright light of microscope (Figures 2(g), (j) and (m)), and then stained with aniline blue to observe callose deposition. The infected rice flower including anthers, stigma and ovary, showed bright aniline blue staining (Figure 2(h)), while the control flower did not exhibit staining, thus, was barely seen under microscope (Figure 2(i)). Many bright aniline blue staining spots were scattered on the bifurcate plumose stigma of the infected rice panicles (Figure 2(k)), in contrast, no bright spots were observed in the stigma from the uninfected plants (Figure 2(l)). To investigate whether callose deposited in the ovary, we cut ovaries longitudinally and stained with aniline blue, the section of the ovary from infected plants showed a bright circle at the 
position of integuments surrounding the ovule (Figure 2(n)), on the contrary, the ovary from the control plants did not show bright circle (Figure 2(o)), indicating that $U$. virens infection can induce callose deposition in the ovary at the place around the ovule.

Booting stage is the critical time for $U$. virens infection. In transverse sections of anthers from $U$. virens infected plants, the pollen mother cells (PMC), both the cell wall and the contents, were slightly stained by aniline blue (Figure 2(p)), in contrast, the PMC from the control plants were heavily stained by aniline blue, indicating large amounts of callose accumulated (Figures 2(q), (s)). Importantly, the PMC of the infected anthers were in a state of plasmolysis (Figure 2(r)), which was probably caused by the metabolites secreted from $U$. virens.

\subsection{U. virens Culture Inhibits Rice Pollen Germination and Pollen Tube Elongation}

$U$. virens secretes compounds including ustiloxins into media, and ustiloxins are toxic to humans and livestock. To verify the effects of secreted compounds on rice pollen development, we used the supernatant of old-cultured $U$. virens to treat rice pollen grains to investigate the germination rate. Rice pollen grains were first treated with germination solution (15\% sucrose, $20 \mathrm{mg} / \mathrm{L}$ borate and $40 \mathrm{mg} / \mathrm{L} \mathrm{CaCl}_{2}$ ), after treatment for $10 \mathrm{~min}$, about $75 \%$ of pollen grains germinated (Figure 3(a)), whereas pollen grain germination was completely inhibited by the treatment of the supernatant (Figure 3(b)). To explore whether or not the supernatant is able to inhibit rice pollen tube elongation, pollen grains were first treated for 6 min in the germination solution, the germinated pollen

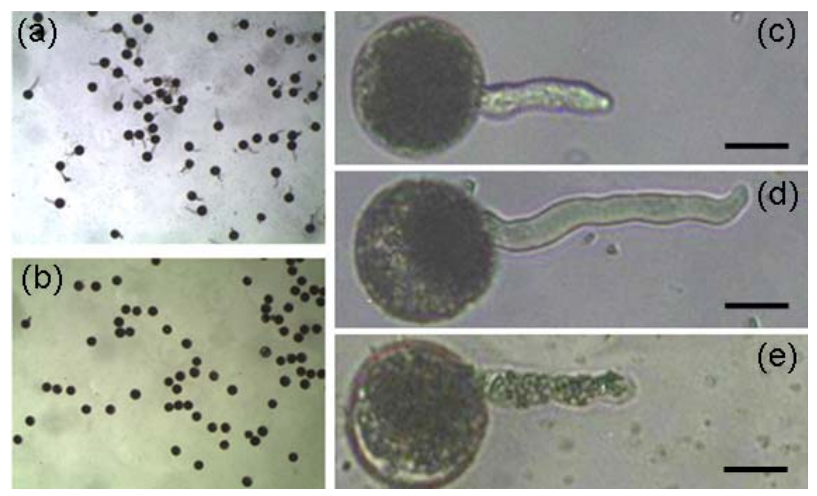

Figure 3. The supernatant of $U$. virens liquid culture inhibits rice pollen grain germination and pollen tube elongation. Scale bars: $20 \mu \mathrm{m}$. (a) Rice pollen grains germinates well in germination solution; (b) None of rice pollen grains germinate in the supernatant of $U$. virens culture; (c) Germinated rice pollen grain showing pollen tube; (d) Rice pollen tube elongates, (e) Rice pollen tube stops elongating after treated with the supernatant of $U$. virens culture. grains were selected and the pollen tube length was recorded (Figure 3(c)). At the same time, some of the selected pollen grains were kept in the germination solution, and others were treated with the supernatant mixture (5 volumes of the supernatant and 1 volume of germination solution), the length of pollen tube was recorded after incubation in the solutions for $9 \mathrm{~min}$. The pollen tube continued to elongate when pollen grains were kept in the germination solution (Figure 3(d)), whereas pollen tube elongation was completely inhibited if the germinated pollens were kept in the supernatant mixture (Figure 3(e)). These data demonstrated that the supernatant of $U$. virens culture can inhibit rice pollen germination and pollen tube elongation, this inhibition is probably due to the ustiloxins secreted by $U$. virens, although we could not rule out the possibility that other compounds existed in the supernatant also have inhibition ability.

\subsection{U. virens Invades Both Pistil and Stamens}

To investigate the routes of $U$. virens infection and analyze the infection effects on rice flower development, we performed semi-thin sectioning of smut balls. The crosssection showed the stigma with two branches were enclosed by hyphae (Figure 4(a)). High magnification showed hyphae could not breach the epidermal cells of the stigma (Figure 4(b)), even the stigma were densely covered by hyphse. In transverse section of smut balls, mycelia compactly packed the stigma and deformed the papillae. Hyphae entered the intercellular spaces of stigma via the thin-walled cells of papillae, they only breached at the sites of the plumose part of the stigma, but could not reach the vascular bundles (Figure 4(c)).

In transverse section of the anthers embedded in the mycelia of smut balls, four locules of an anther were still in shape and visible, but the contents were decomposed and replaced with hyphae (Figure 4(d)). High magnification showed the trace of PMC, but the contents were decomposed and replaced with hyphae (Figure 4(e)). In transverse section of anther filaments embedded in smut balls, the filaments were filled by hyphae but the boundaries between the filaments and the enclosed mycelia were still clear since the hyphae inside the filaments had different staining extent from those surrounding the filaments (Figure 4(f)). Anther filaments consist of epidermis, transmitting and vascular-like tissues. The vascular-like tissue is not a real vascular bundle in comparison to that of the pedicel, thus, it was vulnerable to $U$. virens infection (Figure 4(g)). In high magnification, we were able to verify that the invaded hyphae were well-organized inside the filaments, they decomposed the contents of the cells but did not disturb the original cell organization (Figure 4(h)). These findings are consistent with the results of Tang et al. [11]. 

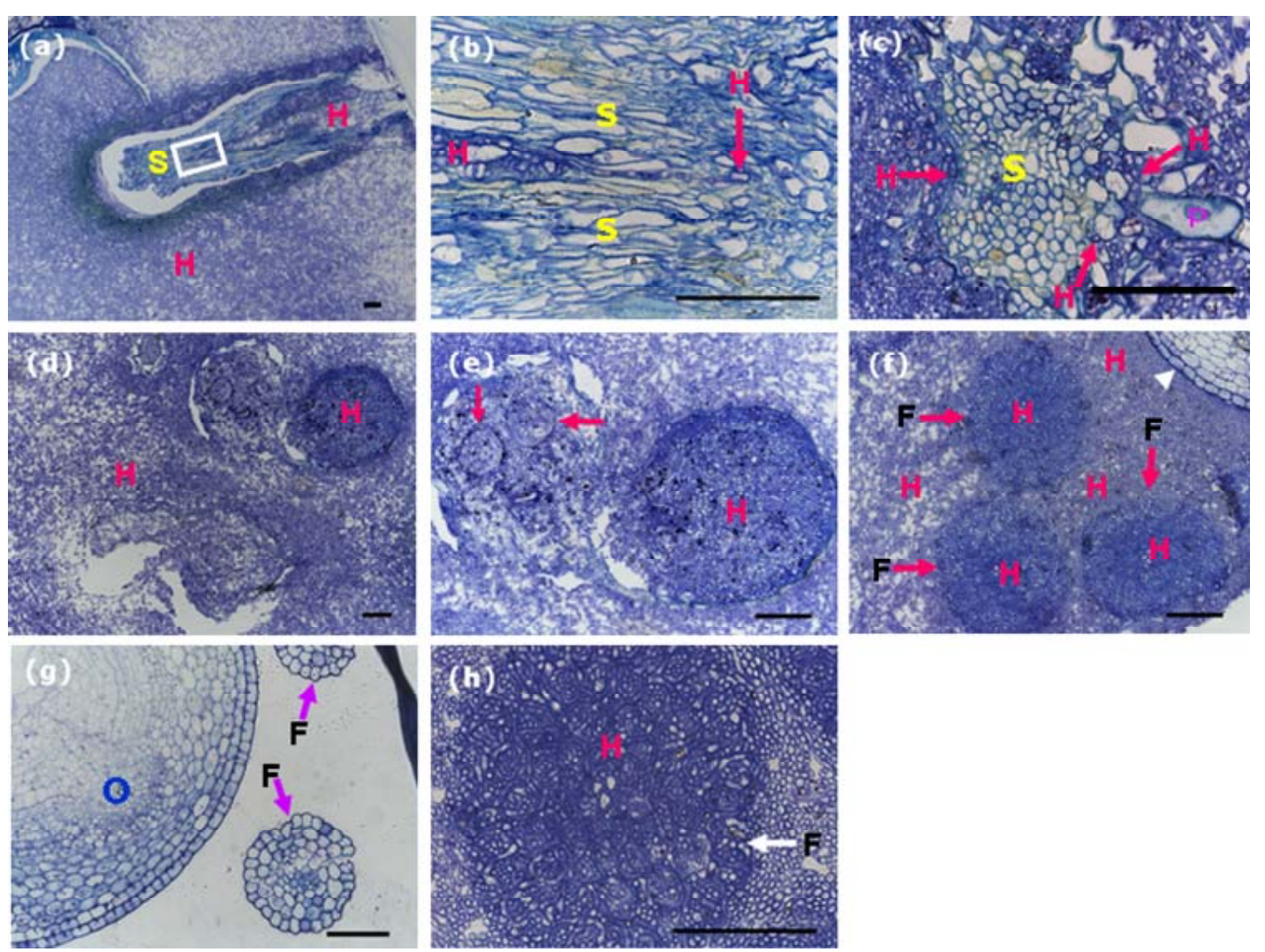

Figure 4. Semi-thin sections of the smut balls reveal hyphae infection of the stamens and stigma. Scale bars: $50 \mu \mathrm{m}$. (a) Transverse section showing bifurcate stigma embedded in mycelia. The white rectangle indicates the converged site of the bifurcate stigma branches; (b) High magnification of the squared region marked in (a), showing hyphae accessed to the bifurcating site, red arrow indicates hyphae; (c) Transverse section of a branch of plumose stigma embedded in mycelia, revealing hyphae in the intercellular spaces of style, arrows indicate hyphae; (d) Transverse section of an anther embedded in mycelia, showing hyphae inside the anther lobules; (e) High magnification of the upper two lobules in (d), arrows representing the traces of residual shape of pollen mother cells, which were dead and full of hyphae; (f) Transverse section of the smut balls, showing anther filaments not only enclosed but also invaded by hyphae, arrows indicate anther filament contour, white arrowhead represents part of the ovary; (g) Transverse section of a rice flower from the control plant showing the structures of ovary and anther filaments, arrows indicate anther filaments; (h) High magnification of an anther filament full of hyphae, arrow indicating anther filament contour. F, filaments; H, hyphae; O, ovary; P, stigma papillae; S, stigma.

\subsection{U. virens Specifically Digests the Integuments and Is Not a Systemic Pathogen}

Rice ovary contains an ovule consisting of the nucellus and an embryo sac containing an egg cell and a central cell. The nucellus is covered by inner and outer integuments, each typically with two layers of cells (Figure 5(a)), and these two layers of the integuments will be absorbed after fertilization.

We did serial cross-sectioning of the smut balls at different positions, in transverse section of mid-region of the ovary persisted in the smut ball and wrapped by mycelia, hyphae were observed in the ovary. The intruded hyphae digested the integuments and grew around the nucellus at the position of integuments without infecting the ovular vascular cells (Figure 5(b)). Although residing the inside of ovary, hyphae did not spread out, they invaded neither the nucellar epidermis nor the inner epidermis of the ovary (Figure 5(b)). On the other hand, the hyphae that wrapped the outside of ovary could not invade the epidermis because we did not observe hyphae existed in the epidermal cells of the ovary, and in high magnification the boundaries between hyphae and rice flower tissues were obviously clear (Figure 5(c)). This result is contradictory to the findings of Tang et al. [11], it is probably caused by different cross-sectioning positions of the ovary in the smut ball.

Rice spikelet is supported by the pedicel that connects to the main stem of the panicle. Transverse section of the pedicel from the control rice plant showed normal vascular bundle (phloem and xylem) and parenchyma (Figure 5(d)). We reasoned that if $U$. virens caused a systemic disease in rice, at least we would observe traces of fungal infection in the pedicel of the spikelet which was 


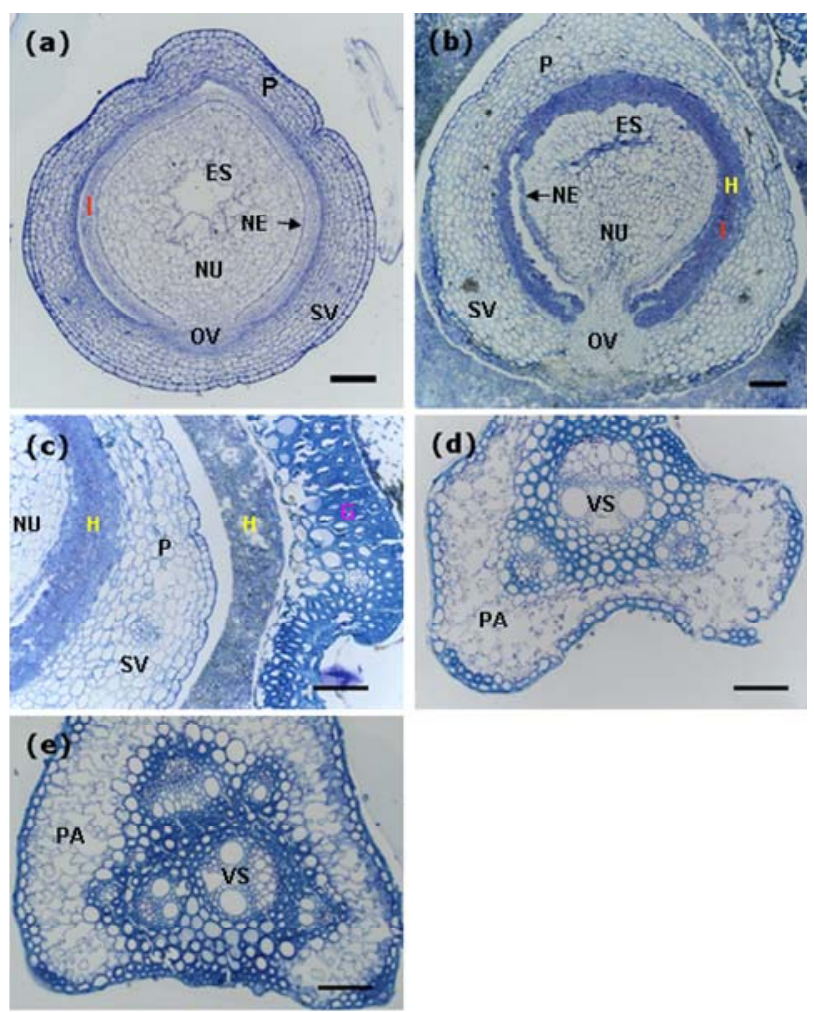

Figure 5. Decomposition of the integument cells of the ovary by invaded hyphae. Scale bars: $50 \mu \mathrm{m}$. (a) Transverse section of an ovary from the control plant, showing components of the ovary; (b) Transverse section of an ovary from the smut ball, showing integument cells (inner and outer layers of cells) decomposed and replaced by the invaded hyphae; (c) Transverse section of the smut balls, showing clear boundaries between the invaded hyphae and the organ tissues; (d) Transverse section of the pedicel from a control spikelet, showing normal structure of the pedicel. (e) Transverse section of the pedicel from a smut ball, revealing vascular system and parenchyma that were as good as those from the control plant. ES, embryo sac; FG, fertile glume; G, glume; H, hyphae; I, integuments (inner and outer integuments); NE, nucellar epidermis; NU, nucellus; OV, ovular vascular trace; $P$, pericarp; PA, parenchyma; SV, stylar vascular trace; VS, vasculature.

infected by $U$. virens and formed smut ball, in this case, some changes to the cells of the pedicel should occur, in other words, hyphae would cause the xylem or phloem tissues of the infected rachilla to become at least partially dysfunctional, and the traces of hyphae should be observed. We carried out cross-sectioning of the pedicel of the spikelet with smut ball at the position just right below the rudimentary glumes. In transverse section, the phloem, xylem and the parenchyma cells were clearly observed and well organized, we did not observe any trace of hyphae in either the vascular tissues or the thin-walled parenchyma cells (Figure 5(e)). Furthermore, there were no differences in cell organization and the state of vascular bundle in the pedicels from the infected and uninfected spikelets. These results indicated that $U$. virens was not able to go through the pedicel from the infected spikelets to the adjacent spikelets, thus, rice false smut is not a systemic disease.

\section{Discussion}

Global agricultural change has brought about many improvements to rice production, including irrigation changes, high-yield variety utilization and heavy application of fertilizers $[3,25]$. Although these changes have led to a significant increase in rice yield, they also provide opportunities to pathogens that may cause new diseases or enhance the severity of the existed diseases. Recently, rice false smut has emerged as one of the major diseases worldwide; its impact on rice production has increased in importance over time and attracted much concern of researchers. However, details of the infection process and the relationship between $U$. virens and rice host are scarce.

The routes of $U$. virens infection have been debated for many years and the infection process of this fungus is still poorly understood. Recently, Ashizawa et al. [24] introduced the green fluorescence protein (GFP) into a $U$. virens strain to monitor the infection process of the pathogen and reported that the infection initiated from the germination of spores on the outer surfaces of spikelets. Hyphae grew on the surfaces and extended to the apex of spikelets, and then colonized onto the inner surfaces of the lemma and palea via the small gap between the lemma and palea. In our experiments, we co-cultured $U$. virens strain and young rice panicles in vitro on medium. Thus, we can monitor the growth of $U$. virens at any time, which provides much convenience to investigate the early process of $U$. virens infection. Our serial observations at different infection time confirmed that hyphae colonize the inner upper spaces of spikelets from the apex and grow downward to infect the pistil and stamens. We did not observe the case in which hyphae infect pistil and stamens first but not appear in the inner upper part of the spikelets.

Colonized inside of the spikelets, $U$. virens hyphae infect pistil and stamens including filaments. Tang et al. [11] reported that hyphae infect filaments and do not penetrate the vascular tissues, which is in consistent with our observations. In addition, we also found that hyphae can not penetrate the epidermis of ovary although large amounts of pathogen hyphae wrapped the whole ovary, which agrees with the findings of Tang et al. [11]. Except for these common observations, there is an important difference between our results and their findings in which we discovered that $U$. virens hyphae enter into the intercellular spaces of style through the junction of the papil- 
lae of stigma, from which hyphae reach the ovary and destroy the integuments of ovule. An open question is how $U$. virens proliferates inside the infected spikelets. We speculate that $U$. virens reaches the inside of the ovary and digests the integuments for the nutrient supply at first, this process would take about $7-8$ days after infection initiation to occur. Ashizawa et al. [24] have reported that 6 days after infection, pathogen can reach the floral organs. Wang [26] reported that the period from the late boot stage to the rupturing stage (i.e. 1 - 4 days before the rupturing stage) is the crucial time for pathogen infection. At the time of 7 - 8 days after pathogen infection, normally, rice plants have completed anthesis, and the ovaries of the uninfected spikelets have been fertilized. After fertilization, the ovary needs large amounts of water, minerals and photosynthesis products to develop. The channel for the ovary to obtain nutriaents is the base of the pedicel connected to the rachis branches. For the infected spikelets, at this time fungal hyphae probably have used up the nutrients from degraded integuments and then, may mimic the way used by the normally fertilized ovary, to obtain nutrients from rice plants. Since large amounts of nutrients are taken by the infected pathogen, the adjacent spikelets may not be able to obtain enough nutrients for their developmental requirement, thus, leading to grain weight reduction and chaffiness. This is not surprising because the integuments are absorbed after ovary fertilization during the developmental process of rice grain. On the other hand, Kessler et al. [27] reported that pollen tube reception and powdery mildew infection share molecular components NTA and FER. These two proteins function either as goddesses of fertility that enable fertilization or as Trojan horses that enable pathogenic fungi to invade plant tissues, although powdery mildew fungi rarely colonize flowers or gain contact with the ovule [28]. It is very likely that $U$. virens mimics the process of ovary fertilization to survive in the infected spikelets although the molecular mechanisms remain unknown.

It is well known that $U$. virens secretes mycotoxins such as ustilaginoidins and ustiloxins [29-31], which are toxic to humans and live stocks. Pathogen infection caused the developmental arrestment and content decomposition of the pollen mother cells. In consequence, no mature pollen grains were produced. Furthermore, the supernatant of pathogen liquid culture inhibited pollen grain germination and suppressed pollen tube elongation. These inhibitory effects are probably due to the functions of the secreted compounds. Previous research reported that ustiloxins are able to not only inhibit tubulin polymerization in a concentration-dependent manner but also induce the depolymerization of preformed microtubules [32]. During the process of infection $U$. virens secretes secondary compounds, which may permeate into cells and destroy cellular cytoskeleton, resulting in destruction of the infected cells and nutrient leakage for pathogen development since there are no special organs such as haustoria found for $U$. virens. Perhaps, only special types of cells at the specific developmental stage are sensitive to the secreted compounds, which may explain the limitation on the infection time of $U$. virens.

Flower-infecting fungi can initiate systemic invasion through the apical meristems. This group of fungi includes many smut fungi that are either seedborne such as Ustilago nuda f.sp. bordei or capable of entering through vegetative tissues such as the anther smut pathogen $\mathrm{Mi}$ crobotryum violaceum [33]. U. virens is able to infect both the stamen and the pistil, and the infection is only restrained to the infected individual spikelets. It did not move from the infected one to the adjacently uninfected ones. The vascular bundle of the pedicel of the smut balls was not infected by hyphae, indicating that $U$. virens is different from those flower-infecting fungi in the way of infection. Our findings support the field investigation because we frequently observed individual smut balls scattered among the spikelets on rice panicle and suggest that rice false smut is not a systemic disease.

\section{Acknowledgements}

This research was supported by grants from the Important Direction Research of Chinese Academy of Sciences Knowledge Innovation Project, no. KSCX2-EW-N-06, and the Hundred Talent Program of Chinese Academy of Sciences to J.X.L.

\section{REFERENCES}

[1] G. S. Deng, "Present Status of Research on False Smut in China," Plant Protection (Chinese), Vol. 15, No. 6, 1989, pp. 39-40.

[2] H. Yaegashi, Y. Fujita and R. Sonoda, "Severe Outbreak of False Smut of Rice in 1988," Plant Protection (Tokyo), Vol. 43, No. 6, 1989, pp. 311-314.

[3] S. Savary, L. Willocquet, F. A. Elazegui, P. S. Teng, P. V. Du, D. F. Zhu, Q. Y. Tang, S. W. Huang, X. Q. Lin, H. M. Sing and R. K. Srivastave, "Rice Pest Constraints in Tropical Asia: Characterization of Injury Profiles in Relation to Production Situation," Plant Disease, Vol. 84, No. 3, 2000, pp. 341-356. http://dx.doi.org/10.1094/PDIS.2000.84.3.341

[4] T. Ashizawa, M. Takahashi, J. Moriwaki and H. Kazuyuki, "Quantification of the Rice False Smut Pathogen Ustilaginoidea virens from Soil in Japan Using RealTime PCR," European Journal of Plant Pathology, Vol. 128 , No. 2, 2010, pp. 221-232. http://dx.doi.org/10.1007/s10658-010-9647-4

[5] W. A. Orton, "Plant Diseases of 1906," Year Book US Department of Agriculture. US Government Printing Of- 
fice, Washington DC, pp. 499-508.

[6] M. C. Rush, A. K. M. Shahjahan and J. P. Jones, "Outbreak of False Smut of Rice in Louisiana," Pant Disease, Vol. 84, No. 1, 2000, p. 100. http://dx.doi.org/10.1094/PDIS.2000.84.1.100D

[7] Y. L. Zhou, Y. J. Pan, X. W. Xie, L. H. Zhu, J. L. Xu, S. Wang and Z. K. Li, "Genetic Diversity of Rice False Smut Fungus, Ustilaginoidea virens and Its Pronounced Differentiation of Populations in North China," Journal of Phytopathology, Vol. 156, No. 9, 2008, pp. 559-564. http://dx.doi.org/10.1111/j.1439-0434.2008.01387.x

[8] H. S. Chib, M. L. Tikoo, C. S. Kalha, B. R. Gupta, S. K. Sing and P. K. Raina, "Effect of False Smut on Yield of Rice," Indian Journal of Mycology and Plant Pathology, Vol. 22, No, 3, 1992, pp. 278-280.

[9] D. H. Lu, X. Q. Yang, J. H. Mao, H. L. Ye, P. Wang, Y. P. Chen, Z. Q. He and F. Chen, "Characterising the Pathogenicity Diversity of Ustilaginoidea virens in Hybrid Rice in China," Journal of Plant Pathology, Vol. 91, No. 2, 2009, pp. 443-451.

[10] T. Ashizawa, M. Takahashi, J. Moriwaki and K. Hirayae, "A Refined Inoculation Method to Evaluate False Smut Resistance in Rice," Journal of General Plant Pathology, Vol. 77, No. 1, pp. 10-16. http://dx.doi.org/10.1007/s10327-010-0279-5

[11] Y. X. Tang, J. Jin, D. W. Hu, M. L. Yong, Y. Xu and L. P. $\mathrm{He}$, "Elucidation of the Infection Process of Ustilaginoidea virens (Teleomorph: Villosiclava virens) in Rice Spikelets," Plant Pathology, Vol. 62, No. 1, 2013, pp. 1-8. http://dx.doi.org/10.1111/j.1365-3059.2012.02629.x

[12] S. T. Chisholm, G. Coaker, B. Day and B. J. Staskawicz, "Host-Microbe Interactions: Shaping the Evolution of the Plant Immune Response," Cell, Vol. 124, No. 4, pp. 803814. http://dx.doi.org/10.1016/j.cell.2006.02.008

[13] J. D. G. Jones and J. L. Dangl, "The Plant Immune System," Nature, Vol. 444, No. 7117, pp. 323-329. http://dx.doi.org/10.1038/nature05286

[14] C. J. Baker and E. W. Orlandi, "Active Oxygen in Plant Pathogenesis," Annual Review of Phytopathology," Vol. 33, 1995, 299-321. http://dx.doi.org/10.1146/annurev.py.33.090195.001503

[15] G. P. Bolwell, L. V. Bindschedler, K. A. Blee, V. S. Butt, D. R. Davies, S. L. Gardner, C. Gerrish and F. Minibayeva, "The Apoplastic Oxidative Burst in Response to Biotic Stress in Plants: A Three-Component System," Journal Experimental Botany, Vol. 53, No. 372, 2002, pp. 1367-1376. http://dx.doi.org/10.1093/jexbot/53.372.1367

[16] R. Desikan, S. J. Neill and J. T. Hancock, "Hydrogen Peroxide-Induced Gene Expression in Arabidopsis thaliana," Free Radical Biology and Medicine, Vol. 28, No. 5, 2000, pp. 773-778. http://dx.doi.org/10.1016/S0891-5849(00)00157-X

[17] I. Apostol, P. F. Heinstein and P. S. Low, "Rapid Stimulation of an Oxidative Burst during Elicitation of Cultured Plant Cells: Role in Defense and Signal Transduction," Plant Physiology, Vol. 90, No. 1, 1989, pp. 109-116. http://dx.doi.org/10.1104/pp.90.1.109
[18] X. Qiu, C. Lei, L. Huang, X. Li, H. Hao, Z. Du, H. Wang, H. Ye, L. Beerhues and B. Liu, "Endogenous Hydrogen Peroxide Is a Key Factor in the Yeast Extract-Induced Activation of Biphenyl Biosynthesis in Cell Cultures of Sorbus aucupari," Planta, Vol. 235, No.1, 2012, pp. 217223. http://dx.doi.org/10.1007/s00425-011-1545-2

[19] A. Daudi, Z. Cheng and J. A. O'Brien, "The Apoplastic Oxidative Burst Peroxidase in Arabidopsis Is a Major Component of Pattern-Triggered Immunity," Plant Cell, Vol. 24, No. 1, 2012, pp. 275-287. http://dx.doi.org/10.1105/tpc.111.093039

[20] J. A. O'Brien, A. Daudi, P. Finch, V. S. Butt, J. P. Whitlegge, P. Souda, F. M. Ausubel and G. P. Bolwell, "A Peroxidase-dependent Apoplastic Oxidative Burst in Cultured Arabidopsis Cells Functions in MAMP-Elicited Defence," Plant Physiology, Vol.158, No. 4, 2012, pp. 2013-2027. http://dx.doi.org/10.1104/pp.111.190140

[21] N. K. Clay, A. M. Adio, C. Denoux, G. Jander and F. M. Ausubel, "Glucosinolate Metabolites Required for an Arabidopsis Innate Immune Response," Science, Vol. 323, No. 5910. pp. 95-101. http://dx.doi.org/10.1126/science.1164627

[22] Y. A. Millet, C. H. Danna, N. K. Clay, W. Songnuan, M. D. Simon, D. Werck-Reichhart and F. M. Ausubel, "Innate Immune Responses Activated in Arabidopsis Roots by Microbe-Associated Molecular Patterns," Plant Cell, Vol. 22, No. 3, 2010, pp. 973-990. http://dx.doi.org/10.1105/tpc.109.069658

[23] M. E. Hood and H. D. Shew, "Applications of KOHAniline Blue Fluorescence in the Study of Plant-Fungal Interactions," Phytopathoglogy, Vol. 86, No. 7, 1996, pp. 704-708. http://dx.doi.org/10.1094/Phyto-86-704

[24] T. Ashizawa, M. Takahashi, M. Arai and T. Arie, "Rice False Smut Pathogen, Ustilaginoidea virens, Invades Through Small Gap at the Apex of a Rice Spikelet Before Heading," Journal of General Plant Pathology, Vol. 78, No. 4, 2012, pp. 255-259. http://dx.doi.org/10.1007/s10327-012-0389-3

[25] K. Dong, B. Chen, Z. Y. Li, Y. Dong and H. L. Wang, “A Characterization of Rice Pests and Quantification of Yield Losses in the Japonica Rice Zone of Yunnan, China," Crop Protection, Vol. 29, No. 6, 2010, pp. 603-611. http://dx.doi.org/10.1016/j.cropro.2010.01.007

[26] G. L. Wang, "Studies on the Infection Period and the Infection Gate of the Chlamydospores of Ustilaginoidea virens (Cooke) Tak. on Rice," Acta Phytophylacica Sinica, Vol. 19, No. 2, 1992, pp. 97-100.

[27] S. A. Kessler, H. Shimosato-Asano and N. F. Keinath, "Conserved Molecular Components for Pollen Tube Reception and Fungal Invasion," Science, Vol. 330, No. 6006, 2010, pp. 968-971.

http://dx.doi.org/10.1126/science.1195211

[28] F. Govers and G. C. Angenent, "Fertility Goddesses as Trojan Horses," Science, Vol. 330, No. 6006, 2010, 922923. http://dx.doi.org/10.1126/science.1198347

[29] K. Koyama and S. Natori, "Further Characterization of Seven bis (naphtha- $\gamma$-pyrone) Congeners of Ustilaginoidins, Pigments of Claviceps virens (Ustilaginoidea virens)," 
Chemical and Pharmaceutical Bulletin, Vol. 36, No. 1, 1988, pp. 146-152.

http://dx.doi.org/10.1248/cpb.36.146

[30] Y. Koiso, Y. Li, S. Iwasaki, K. Hanaoka, T. Kobayashi, R. Sonoda, Y. Fujita, H. Yaegashi and Z. Sato, "Ustiloxins, Antimitotic Cyclic Peptides from False Smut Balls on Rice Panicles Caused by Ustilaginoidea virens," Journal of Antibiotics (Tokyo), Vol. 47, No. 7, 1994, pp. 765-773. http://dx.doi.org/10.7164/antibiotics.47.765

[31] Y. Koiso, N. Morisaki, Y. Yamashita, Y. Mitsui, R. Shirai, Y. Hashimoto and S. Iwasaki, "Isolation and Structure of an Antimitotic Cyclic Peptide, Ustiloxin F: Chemical Interrelation with a Homologous Peptide, Usti- loxin B," Journal of Antibiotics (Tokyo), Vol. 51, No. 4, 1998, pp. 418-422. http://dx.doi.org/10.7164/antibiotics.51.418

[32] Y. Li, Y. Koiso, H. Kobayashi, Y. Hashimoto and S. Iwasaki, "Ustiloxins, New Antimitotic Cyclic Peptides: Interaction with Porcine Brain Tubulin," Biochemical Pharmacology, Vol. 49, No. 10, 1995, pp. 1367-1372. http://dx.doi.org/10.1016/0006-2952(95)00072-8

[33] H. K. Ngugi and H. Scherm, "Biology of Flower-Infecting Fungi," Annual Review of Phytopathology, Vol. 44, 2006, pp. 261-282. http://dx.doi.org/10.1146/annurev.phyto.44.070505.14340 $\underline{5}$ 